\title{
ARTICLE
}

\section{4 nm SERS of NIR Active Hollow Gold Nanotags}

Cite this: DOI: $10.1039 / \times 0 \times x 00000 x$

Received 00th January 2012,

Accepted 00th January 2012

DOI: $10.1039 / x 0 \times x 00000 x$

www.rsc.org/

\author{
H. Kearns, ${ }^{a}$ N. C. Shand, ${ }^{b}$ W. E. Smith, ${ }^{a}$ K. Faulds,${ }^{a}$ and D. Graham, ${ }^{a}$
}

Surface enhanced Raman Scattering (SERS) tags are in situ probes that can provide sensitive and selective probes for optical analysis in biological materials. Engineering tags for use in the near infrared (NIR) region is of particular interest since there is an uncongested spectral window for optical analysis due to the low background absorption and scattering from many molecules. An improved synthesis has resulted in the formation of hollow gold nanoshells (HGNs) with a localised surface plasmon resonance (LSPR) between 800 and $900 \mathrm{~nm}$ which provide effective SERS when excited at $1064 \mathrm{~nm}$. Seven Raman reporters containing aromatic amine or thiol attachment groups were investigated. All were effective but 1, 2-bis(4pyridyl)ethylene (BPE) and 4,4-azopyridine (AZPY) provided the largest enhancement. At approximately monolayer coverage, these two reporters appear to pack with the main axis of the molecule perpendicular or nearly perpendicular to the surface giving strong SERS and thus providing effective $1064 \mathrm{~nm}$ gold SERS nanotags.

\section{Introduction}

Surface enhanced Raman scattering (SERS) is a sensitive analytical technique that can be used to detect molecules attached to or in close proximity to the surface of metal nanoparticles. The enhancements observed are orders of magnitude greater than those reported for conventional Raman scattering, however, the enhancements vary widely depending on analyte and substrate. ${ }^{1,2}$ In the near-infrared (NIR) region of the electromagnetic spectrum the absorption and scattering of many bio-molecules is at a minimum. ${ }^{3}$ Since SERS is dependent mainly on plasmon assisted enhancement rather than the fourth power of frequency, ${ }^{4}$ SERS nanotags that are NIR active can provide sensitive probes with unique spectral fingerprints in this uncongested spectral window. Moreover, with the addition of a $1064 \mathrm{~nm}$ laser excitation there are further SERS benefits such as limited photobleaching, reduced background autofluorescence and greater penetration depth into biological tissues. ${ }^{5,6}$

Designing SERS-active nanostructures with maximum reproducibility and enhancement is a current challenge in bionanotechnology. To date, aggregated noble metal nanoparticles, commonly silver and gold have been used as suspension based SERS substrates, as they are stable materials and have localised surface plasmon resonances (LSPRs) that range from the visible to NIR region. ${ }^{7-8}$ Aggregation of these nanoparticles results in the nanoparticles adhering more closely to one another and creating SERS 'hot spots.' 9 These are created where there are highly localised electromagnetic fields between a number of metal nanoparticles. When a molecule is trapped in this area or in close proximity to the nanoparticle surface, a large electromagnetic enhancement is experienced which results in an intense Raman signal being observed. ${ }^{10}$ However, controlling the enhancement from single aggregates of noble metal nanoparticles in a reproducible manner can be difficult particularly in the NIR. Furthermore, engineering a nanostructure with a consistent size and shape and one that has a tuneable and strong plasmon resonance in the NIR region is still a challenge.

Several SERS substrates have been developed for use at an excitation wavelength of $1064 \mathrm{~nm}$. These include gold nanoshells, ${ }^{1}$ carbon nanotubes, ${ }^{11}$ silver colloid ${ }^{12}$ and gold nanorods. ${ }^{13}$. Unfortunately these nanostructures are normally stabilised in harsher solvents than trisodium citrate (commonly used for HGNs), making further functionalisation more problematic. Halas et al. developed gold coated silica nanoshells that are highly stable, resonant at $1064 \mathrm{~nm}$ and therefore have strong optical properties. ${ }^{1}$ These nanoshells have been modified and employed in a wide range of applications including immunoassays ${ }^{14}$ and photothermal ablation therapy. ${ }^{15}$, ${ }^{16}$ However, the size of these nanoshells are approximately 170 $\mathrm{nm}$ which is problematic for cellular penetration. ${ }^{17}$

Alternatively, 'hollow gold nanoshells' which were first reported by Liang et al. have potential as a SERS substrate for use with $1064 \mathrm{~nm}$ laser excitation. ${ }^{18} \mathrm{HGNs}$ consist of spherical gold shells filled with an embedding medium and the synthesis involves a galvanic replacement reaction of cobalt with gold. ${ }^{19}$ These nanostructures have strong SERS properties ${ }^{20-23}$ and have unique characteristics such as small size (usually from 50-80 $\mathrm{nm}$ ), spherical shape, and a strong tunable plasmon band from the visible to NIR region. ${ }^{19}$ HGNs can be synthesised to be optically active in the NIR region by reducing the inner diameter and wall thickness simultaneously.

Xie et al. reported the synthesis of HGNs with LSPRs from 
650 to $1320 \mathrm{~nm}$ and in the same paper compared the optical and photothermal properties of these nanomaterials. ${ }^{24}$ It was found that only the HGNs with a LSPR of $650 \mathrm{~nm}$ gave a strong SERS signal; with the HGNs with a LSPR of $775 \mathrm{~nm}$ giving very weak SERS signals. Beyond this wavelength no optical response was obtained; therefore suggesting that red-shifted (LSPR $>800 \mathrm{~nm}$ ) HGNs are inactive SERS substrates.

However the work presented here shows that red-shifted HGNs are in fact good SERS substrates at $1064 \mathrm{~nm}$ excitation when the shell thickness is adjusted. Seven reporters using either aromatic amine or thiol groups to attach to the surface were used to label the HGN's, thus developing 'hollow gold nanontags'. In order to obtain effective SERS signals at this excitation wavelength, both these components where required; NIR active HGNs and Raman reporter. Furthermore, the two Raman reporters which gave the largest enhancements and one standard reporter (all commercially available and commonly used with gold nano-substrates) were chosen for further analysis so that the binding and orientation of these reporters on the surface of the HGN could be determined.

\section{Experimental}

Synthesis of HGNs. The HGN synthesis was carried out under inert conditions using a standard Schlenk line to prevent the cobalt nanoparticles from prematurely oxidising. The method described was modified slightly from previous reports. ${ }^{19,24,25}$ In a typical synthesis, cobalt chloride hexahydrate $(200 \mu \mathrm{L}, 0.4 \mathrm{M}$; Fisher Scientific, 99.99\%) and trisodium citrate dihydrate (400 $\mu \mathrm{L}, 0.1 \mathrm{M}$; Sigma-Aldrich, $>99 \%$ ) were added into deionized water $(200 \mathrm{~mL})$ and degassed several times (10 mins vacuum and 15 mins argon). Sodium borohydride ( $1 \mathrm{~mL}, 0.2 \mathrm{M}$; Fisher Scientific, 99\%) was injected into the solution and allowed to react for a further 20 minutes (under constant argon flow) until hydrogen evolution ceased, indicating complete hydrolysis of the reductant. The solution was degassed again (8 min vacuum and $10 \mathrm{~min}$ argon) before chloroauric acid trihydrate $(33 \mathrm{~mL}$, $124 \mu \mathrm{M}$; Fisher Scientific, ACS reagent grade) was injected. This mixture was allowed to react for an additional 10 minutes under argon with vigorous stirring, before being exposed to air, where an obvious colour change from brown to pale green was observed. Finally, trisodium citrate $(1 \mathrm{~mL}, 0.1 \mathrm{M})$ was added to stabilise the hollow gold nanoshell solution. Post synthesis, the HGN solution was concentrated through centrifugation $(4500 \mathrm{x}$ $\mathrm{g}$ ) and the precipitate was re-dispersed in trisodium citrate solution $(2 \mathrm{mM})$ to give a final concentration of $1.37 \mathrm{nM}$ $\left(8.25 \times 10^{11}\right.$ particles/mL).

Characterisation. Investigation into the SERS properties of the hollow gold nanotags were carried out by mixing concentrated HGN solution $(200 \mu \mathrm{L})$ with Raman reporter(s) solution $(15 \mu \mathrm{L}, 10 \mu \mathrm{M})$ and potassium chloride $(150 \mu \mathrm{L}, 30$ $\mathrm{mM}$; Sigma-Aldrich).

The Raman reporters added were 1, 2-bis(4-pyridyl)ethylene (BPE, Sigma-Aldrich >98\%), 4,4-azopyridine (AZPY, SigmaAldrich), 4-mercaptopyridine (MPY, Sigma-Aldrich), 4,4dipyridyl (DP, Sigma-Aldrich), 4-mercaptobenzoic acid (MBA, Sigma-Aldrich), 5-(pyridine-4-yl)-1,3,4-oxadiazole-2-thiol (PYOT, Sigma-Aldrich) and 4-(1H-pyrazol-4-yl)pyridine (PPY, Fluorochem). The Raman measurements were performed using a Real Time Analyzer FT-Raman spectrometer and a laser excitation wavelength of $1064 \mathrm{~nm}$. All the measurements had a 20 second acquisition time and a laser power operating at 420 $\mathrm{mW}$. Each sample was prepared in triplicate and 5 scans of each replicate were recorded. Furthermore, all the Raman spectra have been background corrected.

A Varian Cary 300 Bio UV-Visible Spectrophotometer was used to acquire all the extinction spectra. Scanning electron microscope (SEM) images were obtained using a FEI Sirion 200 ultra-high resolution Schottky field emission scanning electron microscope with FEI software. From the SEM image, both the particle size and shell thickness of the HGNs were confirmed using image-J software. Furthermore, the shell thickness was manually calculated using the average thickness of $100 \mathrm{HGN}$ particles. The SEM samples were prepared on polyelectrolyte functionalised silicon wafers as previously reported. $^{26,20}$ Briefly, silica wafer substrates $(5 \times 5 \mathrm{~mm})$ were washed with methanol wipes and placed in an oxygen plasma cleaner for 90 seconds. Immediately after, they were treated with poly(diallyldimethylammonium chloride) (PDDA ; Sigma Aldrich) solution to create a positive charge on the wafer surface. PDDA solution $(10 \mu \mathrm{L})$ was dissolved in sodium chloride (1 mL, $1 \mathrm{mM}$; Sigma Aldrich, 99\%) and then this solution $(15 \mu \mathrm{L})$ was added dropwise onto the wafer surface. The wafers were left for 20 minutes in a petri dish, before being washed off with water and dried under a stream of nitrogen. Each wafer was then treated with concentrated HGN solution $(50 \mu \mathrm{L})$ and left for a further 10 minutes before being removed and rinsed with water and dried under nitrogen again. This spotting and removal process of the HGN solution was repeated a further twice.

\section{Results and Discussion}

Characterisation of HGNs. The HGN synthesis is based on the galvanic replacement of cobalt with gold, to produce homogenous nanoshells. Initially, a cobalt nanoparticle is synthesised as a template for the growth of HGNs, and by utilising the difference in redox potential between cobalt and gold, it is possible to reduce gold ions while oxidizing the cobalt nanoparticles (CoNPs). The localised surface plasmon resonance of HGNs is controlled by the size of the inner core and the thickness of the gold shell i.e. large inner cores and thin shells produce HGNs with red-shifted LSPRs. It has been previously reported that if the concentration of cobalt chloride and sodium borohydride $\left(\mathrm{NaBH}_{4}\right)$, the sacrificial template and reducing agent respectively, are kept constant then the trisodium citrate capping agent is solely responsible for controlling the core particle size. ${ }^{19}$ By reducing the amount of trisodium citrate present, there are less citrate ions available for stabilising the CoNPs. The small particles aggregate into larger ones resulting in the formation of large inner cores, thus ensuring that the LSPR of the HGNs remain in the NIR region. The thickness of the gold shell can also significantly affect the LSPR of the HGNs i.e. high concentrations of gold $\left(\mathrm{Au}^{3+}\right)$ ions result in thick shells being formed which increases the plasmon oscillation energy resulting in an increase in SERS signal, however it pushes the LSPR towards the visible region. ${ }^{24}$ Therefore an optimum concentration of $124 \mu \mathrm{M}$ chloroauric acid was added and this produced a shell thickness of $\sim 8.8 \mathrm{~nm}$, which was sufficient for the HGNs to be effective SERS substrates. Figure 1, shows the LSPR of the prepared HGNs to be $824 \mathrm{~nm}$ (black line), with the SEM insert clearly showing an image of these unaggregated hollow gold nanoshells. The plasmon resonance frequencies obtained do not match exactly the $1064 \mathrm{~nm}$ excitation frequency but it is has been previously reported that for effective SERS to be achieved, the excitation wavelength does not need to match the wavelength of the 


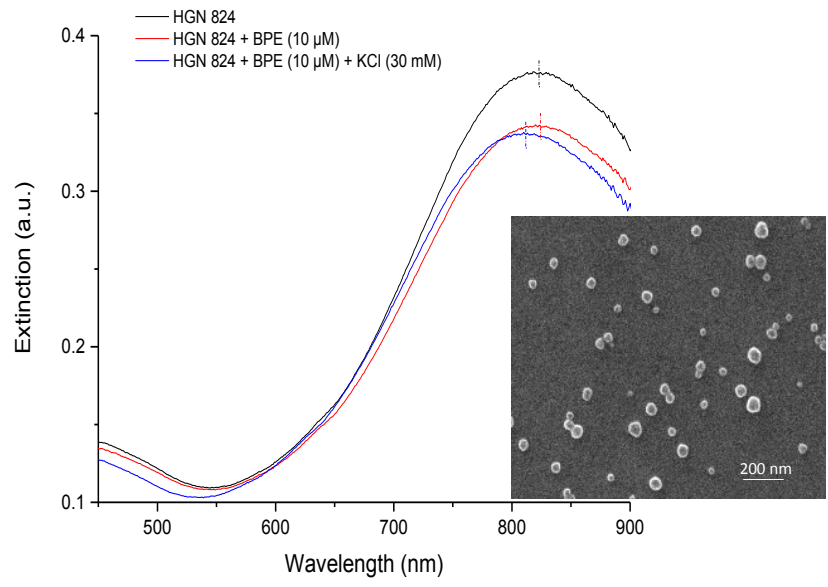

Figure 1 - Extinction spectra showing controlled aggregation caused when $10 \mu \mathrm{M} \mathrm{BPE}$ and $30 \mathrm{mM} \mathrm{KCl}$ salt were added to HGN solutions; black line highlights the hollow gold nanoshells only spectrum; red line is HGN 824 plus the reporter molecule BPE and the blue line is HGN 824 plus BPE and $\mathrm{KCl}$ salt. The blue dotted line clearly shows that a small blue shift in the spectrum occurs with the new SPR recoded at $810 \mathrm{~nm}$. Insert is a SEM image of the HGNs with a LSPR of $824 \mathrm{~nm}$; an average particle size of $78.7 \pm 22.5$ $\mathrm{nm}$ and shell thickness of $8.8 \pm 1.4 \mathrm{~nm}$.

plasmon maximum. ${ }^{27-29}$ Using image $\mathrm{J}$, it was possible to calculate that the average particle diameter was $78.7 \pm 22.5 \mathrm{~nm}$ while the shell thickness was $8.8 \pm 1.34 \mathrm{~nm}$. An SEM of typical HGN's is shown in figure 1.For SERS studies, a Raman reporter concentration of $10 \mu \mathrm{M}$ was used and it can be seen clearly from the extinction spectrum (figure 1), that when 10 $\mu \mathrm{M}$ BPE solution was added to the HGN solution (red line), a dampening of the LSPR band was observed. However the band maximum did not shift in wavelength, indicating that the reporter concentration was low enough that uncontrolled aggregation did not occur, but it was still sufficient to produce intense SERS (see figure 2). When $30 \mathrm{mM}$ potassium chloride, the aggregating agent used in SERS studies later, was added a $14 \mathrm{~nm}$ blue-shift in the maximum was observed, indicating that partial aggregation of the HGNs was occurring. ${ }^{30}$

SERS of hollow gold nanotags with a laser excitation of $1064 \mathrm{~nm}$. The HGNs were functionalised with 7 different Raman reporters and aggregated with potassium chloride $(\mathrm{KCl})$. Initially, the reporters were analysed to see which provided the most intense SERS and following this, three reporters were further characterised.

Figure 2, shows the SERS spectra obtained when each of the reporter molecules at a concentration of $10 \mu \mathrm{M}$ were added to the red-shifted HGNs. All experimental parameters such as HGN concentration, sampling geometries and instrumental parameters including depth of focus, laser power and acquisition times where kept constant for all the hollow gold nanotags tested. Therefore, the reporters were compared based on their relative intensities. The black circle highlights a peak which could be used to identify each reporter. Depending on the reporter, adsorption onto the gold surface can occur via the lone pair electrons of the sulfur or nitrogen atoms or via the conjugated $\pi$-electron system. ${ }^{31}$ Previous studies report that the MPY and MBA bind through the thiol group, whereas BPE, AZPY and DP bind via the nitrogen $(\mathrm{N})$ atom of the pyridyl ring. All are reported as binding with the main axis perpendicular to the surface. ${ }^{31-35}$
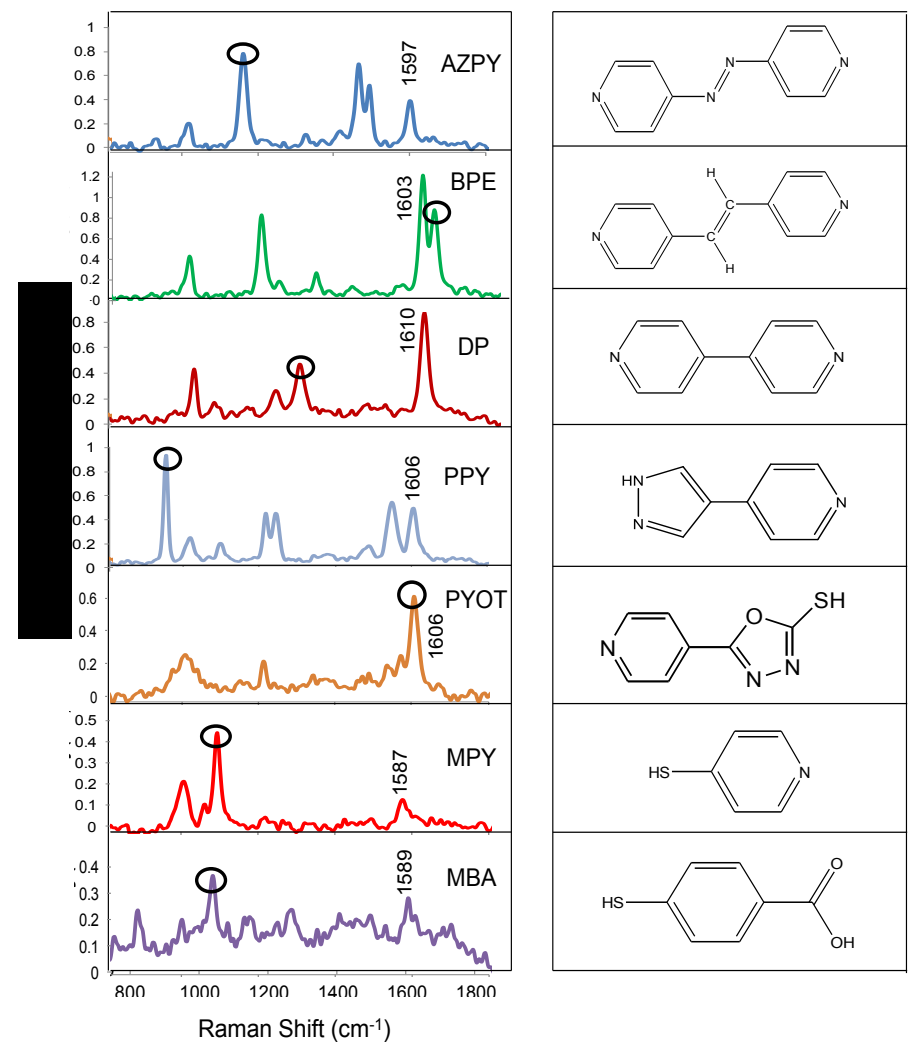

Figure 2 - SERS spectra and structures of all Raman reporters analysed with HGN $824+\mathrm{KCl}$. The circle highlights the characteristic peak which could be used to identify each reporter molecule. A laser excitation of $1064 \mathrm{~nm}$ and an exposure time of 20 seconds were employed. All spectra have been background corrected.

It is expected that PPY will bond to the HGNs via the pyridyl ring $\mathrm{N}$ atom, and PYOT through either the thiol group or the $\mathrm{N}$ atom (of the pyridyl ring) with the softer thiol most likely to bind more strongly. However if effective adsorption is occurring as would be expected, $10 \mu \mathrm{M}$ solutions would lead to about monolayer coverage. This could produce reporterreporter interactions which will stabilise a particular orientation of a reporter by creating a packed surface layer.

The reporter molecules were chosen to bind strongly by chemisorption and consequently when a reporter is adsorbed, there is an overlap between the molecular and metal orbitals resulting in a change in molecular structure and causing some bands to shift and relative intensities to change. ${ }^{33}$ One of the peaks that should be enhanced upon adsorption to metal nanoparticles is the peak at $\sim 1600 \mathrm{~cm}^{-1}$. For reporter's bound through the pyridyl group, this band arises due to the aromatic ring $\mathrm{C}-\mathrm{C} / \mathrm{N}$ stretching. ${ }^{33-34}$ When the surface seeking group from the reporter molecule is in close proximity to the HGN surface and the reporter's adopt an approximately perpendicular orientation it often results in a significant enhancement of this peak being observed It can be seen in figure 2, that intense peaks were observed in the SERS spectra for AZPY, BPE, DP, PPY and PYOT suggesting that these molecules orientated in an upright position on the HGN surface and as such when the laser penetrated the samples a large electromagnetic enhancement was experienced, resulting in the molecules scattering very efficiently and producing strong SERS. 


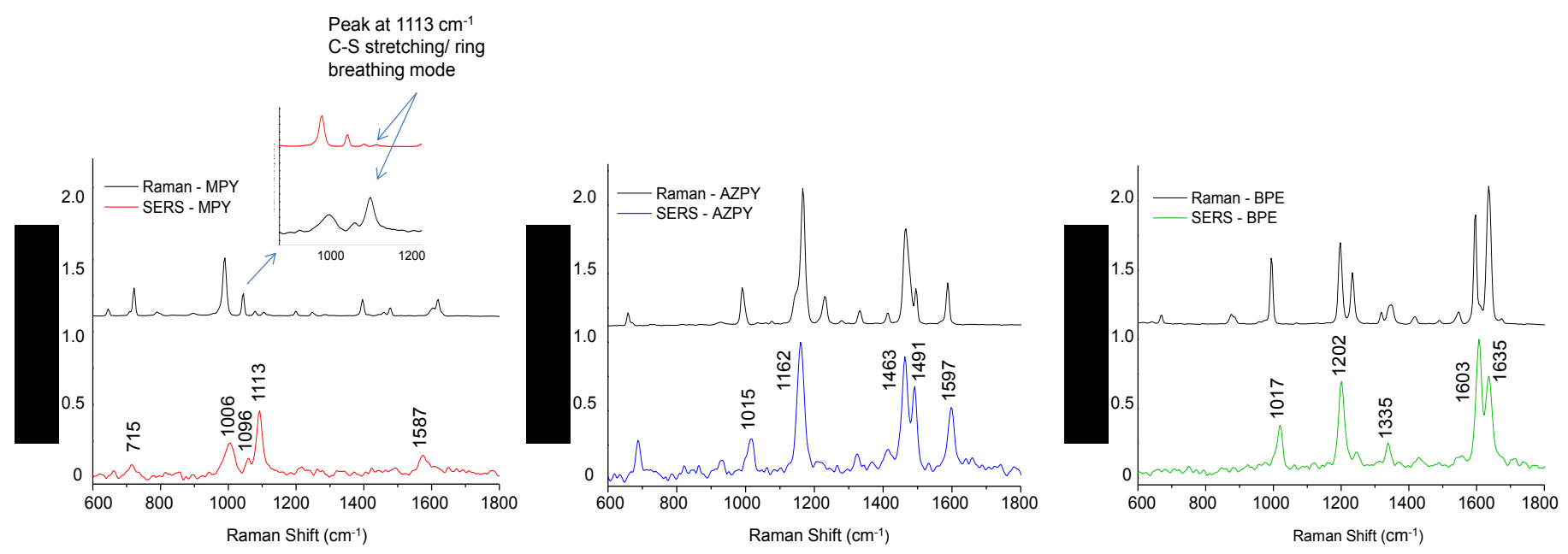

Figure 3 - Comparison of bulk Raman and SERS spectra for each of the Raman reporters. The black lines indicate the bulk Raman spectrum (solid sample) The coloured lines represent the HGN SERS spectrum for each of the reporter molecules at a concentration of $10 \mu \mathrm{M}$ (MPY- red, AZPY, blue and BPE-green) and the insert in the MPY comparison spectra is an enlargement of the important characteristic peaks associated with this molecule. A laser excitation of 1064 $\mathrm{nm}$ and an exposure time of 20 seconds were employed in this analysis. All spectra have been background corrected and normalised to a cyclohexane standard. The Raman spectra were obtained using solid samples, as no spectra could be obtained for any of the reporter bulk solutions at a concentration of $10 \mu \mathrm{M}$.

MPY and MBA give weaker SERS possibly because the layers are packed horizontally. However estimating relative SERS cross sections is very difficult and it may be that they are less polarisable due to the less extended pye systems. Based on these SERS results it can be concluded that the order of performance of these reporters (at $10 \mu \mathrm{M}$ ) as ' 1064 hollow gold nanotags' from best to worst was as follows: BPE $>$ AZPY \& $\mathrm{PPY}>\mathrm{DP}>\mathrm{PYOT}>\mathrm{MPY}>\mathrm{MBA}$. BPE and AZPY have previously been reported to be strong Raman scatterers. ${ }^{24,} 33,34$, 36, 37 They possess highly conjugated structures with their highly delocalised $\pi$-electron systems and two chemically active nitrogen atoms which are ideal for binding to metal surfaces. These molecules can undergo electron transport, intramolecular photoinduced electron transport and energy transfer processes. ${ }^{33,34}$ Therefore, they form the building blocks of many supramolecular systems, have been employed for designing luminescent metal complexes and have been widely utilised as stable reporter molecules for evaluating SERS substrates. ${ }^{24,33} 34,37$

To the best of our knowledge the binding and orientation of these reporter molecules on the surface of a NIR active nanosubstrate have yet to be published. Therefore a comparison study between bulk Raman and $1064 \mathrm{~nm}$ SERS was undertaken for HGNs (LSPR >800 nm) with AZPY, BPE and MPY, three reporters commonly used with gold nano-substrates for SERS analysis (figure 3). ${ }^{37-39}$. Zhuang et al. previously reported the binding and orientation of BPE and AZPY on silver foil at a laser excitation of $1064 \mathrm{~nm} .{ }^{33,34}$ The peaks for MPY binding to silver foils was assigned by $\mathrm{Hu}$ et al. ${ }^{31}$ Due to there being limited assignments and information on how these reporters bind to gold nano-substrates, we have used $\mathrm{Hu}$ and Zhuang's assignments on silver, to help understand and support how these reporter's bind to the gold surface of HGNs. There are a few peak shifts and intensity differences to be noted between the silver and gold SERS data and these will be commented on throughout the discussion as it helps in understanding how these reporters bind and orientate on the HGN surface. For all three reporters, the Raman spectra had to be obtained using solid samples as no spectra could be obtained from the $10 \mu \mathrm{M}$ bulk solution, showing the greater sensitivity of SERS over
Raman for solution based systems. A close examination of the peak shifts and intensities for each reporter molecule was undertaken, however only the main bands which are specific to the binding and orientation on the HGN surface will be discussed here, with their peak assignments being presented in tables $1 \mathrm{a}-\mathrm{c}$. Extended peak assignment tables detailing all the peaks observed in the Raman and SERS spectra for each of the reporter molecules can be found in the supplementary information. Along with further data showing that spherical gold nanoparticles of a similar size produce weaker SERS intensities with these three reporters than the hollow gold nanotags, see figure S1.

Table 1a - Raman shifts $\left(\mathrm{cm}^{-1}\right)$ and peak assignments for AZPY (solid), HGN + AZPY + KCl (SERS - HGN) and Zhuang's silver foil + AZPY (SERS - Ag). ${ }^{33,35,40}$

\begin{tabular}{|c|c|c|c|}
\hline Raman & SERS - HGN & SERS - Ag & Assignments \\
\hline 990 & 1015 & 1010 & Ring Breathing \\
\hline 1167 & 1162 & 1163 & $\begin{array}{l}\text { Ring Breathing, } \\
\mathrm{v}(\mathrm{C}-\mathrm{Nazo}), \delta(\mathrm{C}-\mathrm{H}) \mathrm{py}\end{array}$ \\
\hline 1465 & 1463 & 1466 & $\begin{array}{l}v(C-C) p y, v(C-N) p y \\
\delta(C-H) p y\end{array}$ \\
\hline 1493 & 1491 & 1497 & $\mathrm{v}(\mathrm{C}-\mathrm{C}) \mathrm{py}, \mathrm{v}(\mathrm{N}=\mathrm{N})$ \\
\hline 1587 & 1597 & 1597 & $\begin{array}{l}v(\mathrm{C}-\mathrm{N}) \mathrm{py}, \mathrm{v}(\mathrm{C}-\mathrm{H}) \mathrm{py} \\
\mathrm{v}(\mathrm{N}=\mathrm{N})\end{array}$ \\
\hline
\end{tabular}

$\mathrm{v}=$ stretch, $\delta=$ bend, py = pyridyl ring

For AZPY, the peak at $990 \mathrm{~cm}^{-1}$ in the Raman spectrum assigned as the symmetric ring breathing mode of pyridine and involving a significant displacement of the $\mathrm{N}$ atom of the pyridyl ring, undergoes a $15 \mathrm{~cm}^{-1}$ shift in the SERS spectrum. Furthermore, the peak at $1587 \mathrm{~cm}^{-1}$ also undergoes a shift of 10 $\mathrm{cm}^{-1}$ to appear at $1597 \mathrm{~cm}^{-1}$ in the AZPY SERS spectrum. This band is associated with $v(\mathrm{C}-\mathrm{C}), v(\mathrm{C}-\mathrm{N}), \quad v(\mathrm{~N}=\mathrm{N})$. These modes involve a significant stretch of the pyridyl rings and displacement of the nitrogen along the major axis of the molecule indicating the main interaction with the HGN surface is via the lone pair on this nitrogen atom as is the case for silver. ${ }^{33}$ Furthermore, since these ring breathing modes endured 
the largest displacements it suggests that significant chemisorption occurred.

When a reporter molecule adsorbs onto a metal substrate with a perpendicular orientation there should be a greater enhancement of the $\mathrm{C}-\mathrm{C} / \mathrm{N}$ stretch $\left(\sim 1600 \mathrm{~cm}^{-1}\right)$ over the symmetric ring breathing mode $\left(\sim 1000 \mathrm{~cm}^{-1}\right)$. For AZPY, these peaks occur at 1597 and $1015 \mathrm{~cm}^{-1}$ respectively and it can be seen that indeed the $\mathrm{C}-\mathrm{C} / \mathrm{N}$ stretch is stronger suggesting that AZPY binds with its main axis perpendicular to the HGN surface. In addition, intense azo and ring stretching peaks are observed at 1463 and $1491 \mathrm{~cm}^{-1}$, further suggesting that a vertical orientation is adopted. In general, the HGN SERS spectrum for AZPY is in good agreement with that reported by Zhuang, however, there is one noticeable difference and that is the peak observed at $686 \mathrm{~cm}^{-1}$ in the HGN SERS spectrum is not visible in Zhaung's silver foil spectrum. Peaks below 900 $\mathrm{cm}^{-1}$ are difficult to assign, however, this band could be an SPR enhanced band due the HGNs being resonant in the NIR.

Table 1b - Raman shifts $\left(\mathrm{cm}^{-1}\right)$ and peak assignments for BPE (solid), HGN $+\mathrm{BPE}+\mathrm{KCl}$ (SERS - HGN) and Zhuang's silver foil + BPE (SERS - Ag). ${ }^{34}$, 37,41

\begin{tabular}{llll}
\hline Raman & SERS - HGN & SERS - Ag & Assignments \\
\hline 995 & 1017 & 1011 & Ring Breathing \\
1198 & 1202 & 1205 & v(C-C)py, $\delta(C-N) p y$, \\
& & & $\delta(C-H) p y$ \\
1341 & 1335 & 1329 & $\delta(C-H), \delta(C=C)$ \\
1595 & 1603 & 1600 & $\delta(C-N) p y, \delta(C-H) p y$, \\
& & & v(C-C)py \\
1635 & 1635 & 1635 & (C=C) \\
\hline
\end{tabular}

$v=$ stretch, $\delta=$ bend, py $=$ pyridyl ring

For BPE the ring breathing mode and $\mathrm{C}-\mathrm{N}$ stretching mode of the pyridyl ring behave similarly to the corresponding modes for AZPY suggesting chemisorption occurs once again due to the large displacements being observed. In the SERS spectrum these vibrations undergo a shift of 22 and $7 \mathrm{~cm}^{-1}$ respectively to occur at 1017 and $1603 \mathrm{~cm}^{-1}$ and as stated for the AZPY molecule, involve the movement of the $\mathrm{N}$ atom of the pyridyl ring. The unshifted band at $1635 \mathrm{~cm}^{-1}$ in the SERS spectrum is associated with the vinyl $\mathrm{C}=\mathrm{C}$ stretching mode. If the BPE molecule interacted with the gold (shell) via the $\pi$-electrons, the conjugation between the aromatic ring and vinyl bond would be disrupted, resulting in a peak shift of the vinyl group. Thus, BPE also adsorbs onto the HGN surface via the $\mathrm{N}$ atom of the pyridyl ring and not the $\pi$-bonds. ${ }^{34}$ Furthermore, there is no shift in the $1635 \mathrm{~cm}^{-1}$ peak only a decrease in intensity therefore there is no direct interaction between the vinyl group and HGN surface. In addition, relative to the ring stretching mode at $1602 \mathrm{~cm}^{-1}$, the vinyl stretching band at $1635 \mathrm{~cm}^{-1}$ is weaker in the SERS spectrum than in the Raman spectrum. Zhuang et al using silver foil reported a significant drop in the SERS intensity of this band with $1064 \mathrm{~nm}$ excitation compared to that obtained with shorter wavelength excitation. The authors attributed this to this band being enhanced mainly through interaction with the SPR and not through direct bonding with the silver surface. In this study with a more active NIR plasmon resonance the band is relatively much stronger than in the previous study adding weight to the argument that it is SPR enhanced and not directly involved in surface bonding. This additional evidence all supports the view that BPE binds strongly to the HGN surface through the pyridyl nitrogen's lone pair. Moreover, intense peaks are observed at 1017 and $1603 \mathrm{~cm}^{-1}$ with the $\mathrm{C}-\mathrm{C} / \mathrm{N}$ stretch being significantly stronger than the ring breathing mode indicating that this reporter also binds to the HGN surface with a perpendicular orientation.

Table 1c - Raman shifts $\left(\mathrm{cm}^{-1}\right)$ and peak assignments for MPY (solid), HGN $+\mathrm{MPY}+\mathrm{KCl}$ (SERS - HGN) and Hu's silver foil + MPY (SERS - Ag). ${ }^{31}$

\begin{tabular}{llll}
\hline Raman & SERS - HGN & SERS - Ag & Assignments \\
\hline 721 & 715 & 707 & $\mathrm{v}(\mathrm{C}-\mathrm{S})$, Ring Breathing \\
990 & 1006 & 1010 & Ring Breathing \\
1080 & 1096 & 1061 & $\delta(\mathrm{C}-\mathrm{H})$ py \\
1106 & 1113 & 1098 & $\mathrm{v}(\mathrm{C}-\mathrm{S})$, Ring Breathing \\
1595 & 1587 & 1580 & $\mathrm{v}(\mathrm{C}-\mathrm{C}) \mathrm{py}$ \\
\hline
\end{tabular}

$\mathrm{v}=$ stretch, $\delta=$ bend, py = pyridyl ring

For MPY, the peak at $721 \mathrm{~cm}^{-1}$ in the Raman spectrum associated with ring breathing and C-S stretching undergoes a shift to $715 \mathrm{~cm}^{-1}$ in the SERS spectrum upon adsorption to the HGN surface. Another band which shifts by $7 \mathrm{~cm}^{-1}$ and is significantly enhanced upon adsorption, is the ring-breathing/C-S stretching mode occurring at $1113 \mathrm{~cm}^{-1}$ in the SERS spectrum. The enhancement of this peak can be seen more clearly in the inserted picture (figure 3 ). The band at $1595 \mathrm{~cm}^{-1}$ corresponds to the $\mathrm{C}-\mathrm{C}$ stretching mode and undergoes a shift to $1587 \mathrm{~cm}^{-1}$ in the SERS spectrum. Whereas, the $\mathrm{C}-\mathrm{H}$ and ring breathing modes at 990 and $1080 \mathrm{~cm}^{-1}$ undergo shifts to 1006 and $1096 \mathrm{~cm}^{-1}$ in the SERS spectrum respectively. ${ }^{31,38}$ The peak shifts and intensity changes predominately involve ring breathing modes and $\mathrm{C}-\mathrm{S}$ stretches therefore it is assumed that the MPY molecule will bind to the HGN surface via the sulfur atom. For MPY, the C-C stretch at $1587 \mathrm{~cm}^{-1}$ is slightly weaker than the symmetric ring breathing mode at $1006 \mathrm{~cm}^{-1}$, suggesting that this molecule binds with the main axis parallel to the HGN surface. These results are contrary to $\mathrm{Hu}$ 's as he reported an upright orientation with the C-C stretch appearing stronger (difficult to say for sure as there is no intensity scale). However, the difference in orientation could be due to the thiol binding more strongly to the silver surface than the gold shell of the HGNs or it could be a packing effect of the reporters on the surface. Hu et al. used a reporter concentration of $0.1 \mathrm{mM}$, a factor of 10 greater than in these studies. Both concentrations should give monolayer coverage and this should induce reporter- reporter interactions which will stabilise a particular orientation of a reporter. However, with the higher concentration, it is likely that for effective packing to occur on the surface a vertical orientation will be forced.

These results are in good agreement with previous reports where BPE and AZPY bind to the nanoparticle surface via the $\mathrm{N}$ atom of the pyridyl ring, with a perpendicular or nearly perpendicular orientation being adopted. Whereas MPY binds through the thiol group and depending on the metal substrate and/or concentration of reporter, it may lie flat on the surface as indicted with HGNs or orientate with the main axis perpendicular to the surface as suggested by Hu's silver surface. $^{31}$ Moreover, it is expected that the majority of the Raman reporters tested will adsorb onto the HGN surface in an upright (perpendicular to the surface) orientation, allowing for uniform packing on the HGN surface. ${ }^{31,33,34}$ This perpendicular orientation and the extended $\pi$ system present in these molecules ultimately allows for a greater polarisability change to be experienced when the laser interacts with the sample resulting in the Raman scattering and therefore strong SERS signals being observed at the $1064 \mathrm{~nm}$ excitation wavelength. With the addition of biological targets these unique 1064 SERS 
nanotags could be used in the NIR region for biosensing and 1 labelling of turbid media such as human tissue and blood.

\section{Conclusions}

$1064 \mathrm{~nm}$ SERS detection of NIR active hollow gold nanotags has been demonstrated. An improved synthesis has resulted in the formation of HGNs with a LSPR beyond $800 \mathrm{~nm}$ which provide effective SERS when excited at $1064 \mathrm{~nm}$. There are many advantages of using a $1064 \mathrm{~nm}$ laser and in combination with a good SERS substrate such as these redshifted HGNs, we were able to investigate the interactions occurring between the HGN surface and seven Raman reporters; which contained aromatic amine or thiol attachment groups. All were effective but BPE and AZPY provided the largest enhancement. At approximately monolayer coverage, these molecules pack on the HGN surface with a perpendicular or nearly upright orientation allowing for a greater polarisability to be experienced and as a result strong SERS being obtained at $1064 \mathrm{~nm}$ excitation. Furthermore, the comparison studies between gold and silver led to the understanding that these HGNs are NIR resonant due to the significant enhancement of an SPR attributed C-C stretch at $1600 \mathrm{~cm}^{-1}$ in the HGN spectrum. This band appears very weak in Zhang's silver foil spectrum at $1064 \mathrm{~nm}$ excitation. ${ }^{41}$ Therefore, adding weight to the argument that these HGNs are NIR active and effective candidates for use with a $1064 \mathrm{~nm}$ laser excitation.

Red-shifted HGNs are small (below $100 \mathrm{~nm}$ ) and spherical in shape which means they have the potential to penetrate cellular membranes and tissue cells easily. They are less toxic to cells than silica coated and solid gold nanoparticles ${ }^{42}$ and are stable in sodium citrate allowing for easier functionalisation of the outer shells. A combination of these unique physical properties of the HGNs and the demonstration of the great SERS capabilities make hollow gold nanotags with LSPRs $>800 \mathrm{~nm}$ potential candidates for future use in cellular and human tissue imaging, where $1064 \mathrm{~nm}$ lasers are already widely employed.

\section{Acknowledgements}

This work was supported by Dstl and the Engineering and Physical Sciences Research Council [grant number $\mathrm{EP} / \mathrm{J} 500550 / 1]$. The research data associated with this paper will become available at the following link from Jan 2015 https://pure.strath.ac.uk/portal/en/projects/epsrc-doctoraltraining-grant(288fce07-0618-470d-8f27-af9f3db358ea).html DG acknowledges the Royal Society for support in the form of a Wolfson Research Merit Award.

\section{Notes and References}

${ }^{a}$ Centre for Molecular Nanometrology, West CHEM, Department of Pure and Applied Chemistry, University of Strathclyde, 295 Cathedral St., Glasgow, G1 1XL, UK.

E-mail: Duncan.graham@strath.ac.uk; Tel: +44 (0)141 5484701

${ }^{b}$ Dstl, Porton Down, Salisbury, UK

Electronic Supplementary Information (ESI) available: [details of any supplementary information available should be included here]. See DOI: 10.1039/b000000x/
S. J. Oldenburg, S. L. Westcott, R. D. Averitt and N. J. Halas, The Journal of chemical physics, 1999, 111, 47294735.

2. Y. Wang and S. Schlücker, Analyst, 2013, 138, 2224-2238.

3. G. Ku, M. Zhou, S. Song, Q. Huang, J. Hazle and C. Li, Acs Nano, 2012, 6, 7489-7496.

4. E. Smith, G. Dent and J. Wiley, Modern Raman spectroscopy: a practical approach, Wiley Online Library, 2005.

5. A. Bashkatov, E. Genina, V. Kochubey and V. Tuchin, Journal of Physics D: Applied Physics, 2005, 38, 2543.

6. M. Culha, B. Cullum, N. Lavrik and C. K. Klutse, Journal of Nanotechnology, 2012, 2012.

7. M. Rycenga, Z. Wang, E. Gordon, C. M. Cobley, A. G. Schwartz, C. S. Lo and Y. Xia, Angewandte Chemie International Edition, 2009, 48, 9924-9927.

8. D. A. Giljohann, D. S. Seferos, W. L. Daniel, M. D. Massich, P. C. Patel and C. A. Mirkin, Angewandte Chemie International Edition, 2010, 49, 3280-3294.

9. I. A. Larmour, K. Faulds and D. Graham, The Journal of Physical Chemistry C, 2010.

10. S. L. Kleinman, R. R. Frontiera, A.-I. Henry, J. A. Dieringer and R. P. Van Duyne, Physical Chemistry Chemical Physics, 2013, 15, 21-36

11. S. Lefrant, I. Baltog, M. Baibarac, J. Mevellec and O. Chauvet, Carbon, 2002, 40, 2201-2211.

12. N. Leopold and B. Lendl, The Journal of Physical Chemistry $B, 2003,107,5723-5727$.

13. B. Nikoobakht and M. A. El-Sayed, Chemistry of Materials, 2003, 15, 1957-1962.

14. L. Hirsch, J. Jackson, A. Lee, N. Halas and J. West, Analytical Chemistry, 2003, 75, 2377-2381.

15. L. R. Hirsch, R. Stafford, J. Bankson, S. Sershen, B. Rivera, R. Price, J. Hazle, N. Halas and J. West, Proceedings of the National Academy of Sciences, 2003, 100, 13549-13554.

$16 . \quad$ D. P. O'Neal, L. R. Hirsch, N. J. Halas, J. D. Payne and J. L. West, Cancer letters, 2004, 209, 171-176.

17. Y. Xia, W. Li, C. M. Cobley, J. Chen, X. Xia, Q. Zhang, M. Yang, E. C. Cho and P. K. Brown, Accounts of chemical research, 2011.

18. H. P. Liang, L. J. Wan, C. L. Bai and L. Jiang, The Journal of Physical Chemistry B, 2005, 109, 7795-7800.

19. A. M. Schwartzberg, T. Y. Olson, C. E. Talley and J. Z. Zhang, The Journal of Physical Chemistry B, 2006, 110, 19935-19944.

20. H.-n. Xie, I. A. Larmour, K. Faulds and D. Graham, The Journal of Physical Chemistry C, 2012.

21. E. Boisselier and D. Astruc, Chem. Soc. Rev., 2009, 38, 1759-1782.

22. M. C. Daniel and D. Astruc, Chemical Reviews-Columbus, 2004, 104, 293.

23. H. Chon, C. Lim, S.-M. Ha, Y. Ahn, E. K. Lee, S.-I. Chang, G. H. Seong and J. Choo, Analytical chemistry, 2010, 82, 5290-5295.

24. H.-n. Xie, I. A. Larmour, Y.-C. Chen, A. W. Wark, V. Tileli, D. W. McComb, K. Faulds and D. Graham, Nanoscale, 2013, 5, 765-771.

25. S. Preciado-Flores, D. Wang, D. A. Wheeler, R. Newhouse, J. K. Hensel, A. Schwartzberg, L. Wang, J. Zhu, M. BarbozaFlores and J. Z. Zhang, J. Mater. Chem., 2010.

26. A. McLintock, N. Hunt and A. W. Wark, Chem. Commun., 2011, 47, 3757-3759.

S. Abalde-Cela, S. Ho, B. Rodríguez-González, M. A. Correa-Duarte, R. A. Álvarez-Puebla, L. M. Liz-Marzán and N. A. Kotov, Angew. Chem., 2009, 121, 5430-5433.

28. B. Nikoobakht, J. Wang and M. A. El-Sayed, Chemical Physics Letters, 2002, 366, 17-23.

29. I. Khan, D. Cunningham, S. Lazar, D. Graham, W. Ewen Smith and D. W. McComb, Faraday Discuss., 2006, 132, 171-178.

30. M. P. Melancon, W. Lu, Z. Yang, R. Zhang, Z. Cheng, A. M. Elliot, J. Stafford, T. Olson, J. Z. Zhang and C. Li, Molecular cancer therapeutics, 2008, 7, 1730. 
31. J. Hu, B. Zhao, W. Xu, B. Li and Y. Fan, Spectrochimica Acta Part A: Molecular and Biomolecular Spectroscopy, 2002, 58, 2827-2834.

32. A. Michota and J. Bukowska, Journal of Raman Spectroscopy, 2003, 34, 21-25.

33. Z. Zhuang, J. Cheng, X. Wang, Y. Yin, G. Chen, B. Zhao, H. Zhang and G. Zhang, Journal of molecular structure, 2006, 794, 77-82.

34. Z. Zhuang, J. Cheng, H. Jia, J. Zeng, X. Han, B. Zhao, H. Zhang, G. Zhang and W. Zhao, Vibrational spectroscopy, 2007, 43, 306-312.

35. X. Wang, B. Zhao, Y. Wang, Y. Wu, W. Xu and Y. Fan, Materials Science and Engineering: C, 1999, 10, 3-6.

36. W. h. Yang, J. Hulteen, G. C. Schatz and R. P. Van Duyne, The Journal of chemical physics, 1996, 104, 4313-4323.

37. A. Kim, F. S. Ou, D. A. Ohlberg, M. Hu, R. S. Williams and $\mathrm{Z}$. Li, Journal of the American Chemical Society, 2011, 133, 8234-8239.

38. T. Lou, Y. Wang, J. Li, H. Peng, H. Xiong and L. Chen, Analytical and bioanalytical chemistry, 2011, 401, 333-338.

39. W. E. Doering, M. E. Piotti, M. J. Natan and R. G. Freeman, Advanced Materials, 2007, 19, 3100-3108.

40. Z. Zhuang, X. Shang, X. Wang, W. Ruan and B. Zhao, Spectrochimica Acta Part A: Molecular and Biomolecular Spectroscopy, 2009, 72, 954-958.

41. Z. Zhuang, X. Shi, Y. Chen and M. Zuo, Spectrochimica Acta Part A: Molecular and Biomolecular Spectroscopy, 2011, 79, 1593-1599.

42. D. Sikdar, I. D. Rukhlenko, W. Cheng and M. Premaratne, Biomedical optics express, 2013, 4, 15-31. 